\title{
LES NORMES DANS LEUR DIVERSITÉ. QUELLE PERTINENCE ?
}

\author{
Colette FEUILLARD \\ Université Paris Descartes/COMUE Paris-Sorbonne-Cité
}

\begin{abstract}
En): The concept of norm is particularly ambiguous in French. It can refer to official, objective, subjective, regional, popular standards, etc. Therefore the objective of this paper is to determine if these different standards have almost the same status and the same functioning or if it would be better to apply this notion only to the official language by providing it with a relevant scientific value.
\end{abstract}

Keywords (En): Norm; use; codification; standardization; relevance.

La notion de norme n'est pas propre aux sciences du langage. On la retrouve en philosophie, en sociologie, dans le monde industriel, etc. Cet exposé, cependant, sera centré exclusivement sur la linguistique, même si à certains moments seront évoquées d'autres approches afin de mieux mettre en lumière la spécificité de la problématique dans le traitement des faits de langue. En linguistique, comme dans d'autres domaines, cette notion a donné lieu à de très nombreux débats et à une grande diversité d'emplois, de telle sorte qu'aujourd'hui le terme générique LA NORME a fait place à une pluralité de normes (officielle, standard, dialectale, régionale, populaire, sociale, individuelle, objective, subjective, etc.) résultant de la multiplicité des points de vue et des domaines pris en compte par les descripteurs dans l'analyse des données langagières. Ces nombreuses subdivisions tendent à obscurcir la notion première de norme et à en faire un concept «surchargé» pour reprendre l'expression de Bergson. Cette polyvalence, terme préféré à celui de polysémie dans la mesure où ce qui est en jeu, ici, n'est pas la pluralité sémantique initiale du terme, mais son application à des champs de plus en plus diversifiés, me paraît entraîner une dilution du concept en tant qu' ' outil descriptif », et poser de ce fait le problème de sa validité scientifique et de sa pertinence actuelle en linguistique. En effet, pour qu'une notion soit véritablement opératoire, elle devrait avoir un sens univoque et ne s'appliquer qu'à des objets présentant des propriétés identiques de manière à circonscrire et à unifier le domaine auquel elle s'applique.

Or, il me semble que l'extension d'emploi du terme norme répond plus à une appréhension intuitive de cette notion qu'à une approche scientifique, ce qui risque d'occulter sa spécificité initiale, si tant est qu'elle n'ait jamais été explicitement établie. C'est sur ces différents points que portera l'étude qui va suivre, l'objectif étant de voir s'il ne serait pas possible de lui conférer une pertinence qui lui attribuerait un caractère scientifique dénué de toute ambiguïté.

Après avoir rappelé brièvement l'origine de ce concept et ses caractéristiques dans des emplois autres que linguistique, j'examinerai la manière dont la norme linguistique s'est constituée en français puis en tchèque. Il convient, en effet, de déterminer le statut propre de chacune de ces normes et de dégager, dans la mesure du possible, les critères qui ont présidé à leur instauration respective, afin de voir 
si ceux-ci répondent à des principes généraux ou s'ils sont étroitement liés à la situation historico-politique, culturelle et linguistique des pays concernés. Puis je présenterai succinctement quelques-unes des acceptions prises par cette notion chez certains linguistes afin de déterminer ou non un éventuel consensus entre eux.

Dans le cadre d'un exposé aussi limité que celui-ci, il ne me sera pas possible de prendre en compte des normes ${ }^{1}$ en cours d'élaboration avec toutes les implications politiques, économiques, sociales, linguistiques qu'elles supposent (cf. en Afrique, par exemple), ou les normes auxquelles il est fait référence dans des pays où l'unité politique et linguistique s'est faite de manière relativement récente (Italie, Allemagne, etc.). En effet, la forme prise par ces normes et leurs conditions d'emploi sont largement déterminées par le contexte général (sociopolitique, linguistique, etc.) dans lequel elles ont vu le jour. Les seules langues envisagées seront le français de l'Hexagone et le tchèque, compte tenu du contexte $^{2}$ dans lequel se situe cette étude.

Il en va de même des normes qui dépassent le champ linguistique tout en l'incluant mais qui impliquent l'emploi de critères spécifiques compte tenu de l'objet envisagé et de l'objectif visé, telle la norme esthétique de Mukařovský. Toutefois, il convient de préciser que celle-ci s'inscrit dans le cadre de la réflexion générale du Cercle linguistique de Prague sur la norme et sa codification, car elle repose sur les principes structuralistes et fonctionnalistes qui fondent la théorie développée par le CLP.

Par ailleurs, les normes communicationnelles et pragmatiques ne seront pas examinées, bien qu'elles soient en interaction avec celles qui seront présentées. Enfin, ne pourront être abordés ni les rapports entre norme et enseignement, ni des écarts par rapport à la norme, ou plus largement de la « correction » ou de «l'incorrection » des productions langagières, avec toutes les réserves que doivent impliquer ces caractérisations.

Chacun des aspects évoqués, tout en étant essentiels dans la constitution et dans l'évolution de «la » norme, voire dans son adaptation, ne peuvent être traités que dans le cadre d'une recherche spécifique centrée sur la problématique concernée, qui n'est pas celle de l'étude proposée, cette dernière étant circonscrite à la conception théorique de la notion de norme et à son éventuelle validité scientifique.

\section{Origine de la notion}

Du point de vue étymologique, le terme norme vient du latin norma, issu luimême du grec gnomon. Il signifie «équerre », instrument qui sert à tracer un angle droit ou une ligne droite. Gnomon renvoie, en outre, à la « faculté de connaître », au « bon sens », à la «droite raison» (BLAY, 2007 : 567-568). La norme acquiert ainsi un double statut, celui d'instrument et celui de modèle, ce qui lui confère une

\footnotetext{
${ }^{1}$ Dans ce passage, l'emploi du pluriel de norme renvoie au «standard » de chaque communauté linguistique envisagée. Il est donc l'équivalent du singulier; il ne s'agit nullement des divers types d'usage en cours au sein de celle-ci.

${ }^{2}$ Cf. Réseau Théories et concepts du cercle linguistique de Prague au seuil du XXI siècle qui, à côté de linguistes tchèques, regroupe des chercheurs français.
} 
double valeur, objective lorsqu'elle désigne l'outil, subjective quand elle fait référence au devoir, à la raison. C'est à ce titre que l'équerre est devenue l'un des symboles des Francs-maçons.

\section{La norme non linguistique}

Il s'agit d'une notion transversale qui, comme cela a été signalé précédemment, n'est pas propre à la linguistique ; elle est présente dans la quasitotalité des domaines de la vie quotidienne, juridique, scientifique, économique, technique, industriel, médical, sanitaire, philosophique, éthique, artistique, comportemental (savoir-vivre), etc. En dépit de cette diversité des usages se manifestent néanmoins des propriétés communes. La question est alors de savoir si elles sont constitutives de la norme elle-même. Pour les dégager, je me référerai aux domaines de la technique à laquelle est associée la sécurité (construction d'un échafaudage), de l'industrie (construction des nouveaux $\mathrm{TER}^{3}$ ), et à une norme sociale, telle que le savoir-vivre (dresser une table) :

Ces normes mettent en jeu les facteurs suivants :

1/ elles proviennent de l'intervention d'une entité extérieure (institution, organisme, sous-groupes d'individus, individu ${ }^{4}$ ) qui édicte la norme par rapport à l'objet en question. Il s'agit donc d'une construction fondée sur des hypothèses.

$2 /$ elles exigent une sélection d'éléments internes ou externes à l'objet, ce qui implique nécessairement une projection des concepteurs à la fois sur la nature de l'objet et sur la finalité de la norme à instaurer. La sélection n'est, de ce fait, jamais purement objective.

Il en découle :

3/ une individuation de l'objet qui acquiert au travers de la norme une identité propre.

4/ une valeur fonctionnelle assignée à cet objet par les promoteurs de la norme, cette dernière leur servant d'instrument de régulation. La fonctionnalité de la norme n'est pas inhérente à celle-ci, mais à sa mise en pratique, c'est-à-dire à son application; elle est liée à une finalité particulière, posée préalablement par les concepteurs.

5/ En conséquence, elle n'est pas en soi absolument impérative, car l'on peut toujours estimer qu'une autre norme aurait été en mesure de produire les mêmes effets. Cela vaut, par exemple, pour les normes industrielles et en particulier pour les normes sociales de type comportemental.

L'objectif visé est généralement multiple. Il peut concerner :

6/ la protection des différents acteurs impliqués par la norme établie vis-à-vis de la concurrence (usager, concepteur et réalisateur, produit). Cette protection est parfois simplement supposée, comme c'est le cas des normes de bienséance, un non-respect de ces dernières pouvant entraîner une stigmatisation.

Cela a pour conséquence :

\footnotetext{
${ }^{3} \mathrm{TER}=$ transport express régional, en l'occurrence train.

${ }^{4}$ Parmi les normes mentionnées ici, seules les normes de savoir-vivre ne sont pas constituées par un véritable groupe.
} 
6/ Une valorisation du produit, qui peut être afférente à la qualité (normes sanitaires, la moralité (protection), l'esthétique (normes picturales), etc., ces aspects n'étant pas nécessairement exclusifs les uns des autres.

Une norme, dès l'instant où elle est posée, est nécessairement formelle, quelle que soit sa nature, dans la mesure où elle est fondée sur un ensemble de règles. En revanche, elle peut conduire, selon Durkheim, à des «sanctions positives, telles les récompenses, honneurs ou titres [qui] favorisent certains comportements. Les sanctions négatives les condamnent, soit de façon explicite du fait de l'application du droit pénal [par exemple], soit de façon implicite, par la réprobation, l'évitement, la moquerie, etc. » (PILlON, 2003 : 11).

Certaines de ces normes peuvent à première vue être considérées comme objectives du fait qu'elles s'appliquent à des éléments concrets de la réalité (cf. les normes industrielles). Cependant, elles font nécessairement intervenir des facteurs subjectifs, dans la mesure où elles sont le résultat d'un choix de la part de ceux qui les ont établies et présupposent une valorisation. D'autres, en revanche, sont exclusivement subjectives comme les normes censées dicter le savoir-vivre. Ces dernières ne sauraient répondre au principe de qualité, puisqu'aucun critère ne permet de le vérifier. On pourrait d'ailleurs le remettre en cause pour des normes supposées objectives, étant donné que rien ne garantit leur supériorité par rapport à d'autres, susceptibles d'être équivalentes. En d'autres termes, la distinction que l'on peut faire entre normes objectives et normes subjectives ne doit pas être dichotomique. Elle se situe dans un continuum marqué par des degrés, qui vont du plus objectif (normes industrielles) au plus, voire totalement subjectif (cf. les hypothétiques normes de savoir-vivre).

Les normes instaurées ont un caractère social et en principe, une validité générale pour les membres auxquels elles s'adressent et non par rapport à un seul individu. Elles permettent ainsi de délimiter un sous-groupe au sein d'une collectivité, ce qui peut entraîner, selon les domaines concernés, un sentiment d'appartenance identitaire (cf. normes esthétiques, de savoir-vivre, etc.) ou à l'inverse un sentiment de stigmatisation (cf. normes de savoir-vivre), voire d'exclusion chez ceux qui ne les partagent pas. Ce non-respect des normes peut également exprimer une volonté de transgression à des fins identitaires ou de révolte contre la société.

Ces normes sont, dans certains cas, absolument nécessaires et impératives, et toute absence de norme ou tout manquement aux règles émises peut avoir une incidence négative (cf. le problème de la dimension des nouveaux TER en France qui ne s'adapte pas à celle de certains quais de gare, d'où la nécessité de remettre à niveau ces derniers).

Enfin, les normes ne sont pas universelles. Elles varient selon les pays. Fixes à un moment donné, elles sont aussi évolutives dans le temps, car elles sont partiellement déterminées par les modifications des savoirs, des techniques, etc. 


\section{La norme linguistique}

\subsection{Constitution d'une norme « officielle » en français et en tchèque ${ }^{5}$}

Il est impossible d'aborder cette problématique sans rappeler très brièvement l'histoire de ces deux langues en les resituant dans leur contexte respectif. Cependant concevoir le français ou le tchèque comme des entités bien définies et clairement délimitées correspond à une abstraction face à la diversité de leurs réalisations. Néanmoins, il s'est avéré indispensable à un moment donné de proposer pour chacune d'elles une norme de référence que je qualifierai provisoirement de «officielle », bien que pour le tchèque on parle plutôt de norme standard ${ }^{6}$.

\subsubsection{La norme du français}

Le français «officiel», tel qu'il se présente actuellement, s'est progressivement constitué au fil du temps. Issu essentiellement du latin populaire, il a longtemps coexisté avec les dialectes d'oïl au Nord de la France et les dialectes d'oc au Sud. Ces derniers se développèrent jusqu'à l'avènement des Capétiens en l'absence d'un pouvoir central lié à la structure féodale du pays. Les grands moments de sa formation, de son officialisation et de sa diffusion ont été les suivants :

a) La rédaction des Serments de Strasbourg (842). Il s'agissait d'un texte juridique, l'une des premières attestations de la langue sous sa forme écrite.

b) L'invention de l'imprimerie (1438-fin du $\mathrm{XV}^{\mathrm{e}}$ ). Elle permit la fixation et la diffusion des textes écrits.

c) la promulgation, à la Renaissance, de l'Ordonnancede Villers-Cotterêts (1539) par François I ${ }^{\text {er }}$. Elle conféra définitivement au français sa légitimité et en fit la langue officielle du droit et de l'administration. Cela contribua à un mouvement de centralisation linguistique mais aussi politique, en confortant l'autorité du Roi, qui se renforça plus tard sous Louis XIV.

d) L'intervention d'écrivains, de grammairiens, de savants, avec l'élaboration de grammaires et de dictionnaires : deux tendances alors se dessinèrent, l'une, bien que centrée sur la littérature, restait ouverte à d'autres usages, souvent plus populaires, l'autre se voulait puriste et cherchait à respecter la tradition. Le premier mouvement était représenté tout d'abord par les auteurs de la Pléiade, notamment avec Ronsard, Du Bellay et sa Défense et illustration de la langue française (1549) ainsi que par certains grammairiens tels que Ménage, puis plus tard par Molière, Scarron, Balzac, Hugo, Sue, Zola, etc. Parallèlement se développa un courant puriste, dont le chef de file fut au départ Malherbe et à sa suite Vaugelas avec Remarques sur la langue française, utiles à ceux qui veulent bien parler et bien écrire (1647), puis, dans la même lignée, Bouhours. La Grammaire générale et raisonnée de Port-Royal (1660) d'Arnauld et Lancelot fut l'une des toutes premières contributions à la formalisation de la langue et à son intellectualisation.

\footnotetext{
${ }^{5}$ Seuls les points saillants de l'histoire respective de chacune de ces normes seront présentés.

${ }^{6}$ Cette question sera débattue ultérieurement.
} 
À côté du pouvoir politique et des Lettrés qui continuèrent à jouer un rôle essentiel dans la forme que prit le français «officiel» jusqu'à aujourd'hui, deux autres facteurs contribuèrent à le façonner, la mise en place d'institutions ainsi que la généralisation de l'enseignement.

Parmi ces institutions, il y eut tout d'abord

e) La création de l'Académie française par Richelieu en 1635 ,

f) et plus récemment, au $\mathrm{XX}^{\mathrm{e}}$ siècle, celle du Haut Comité pour la langue française, qui vit le jour grâce au Général de Gaulle en 1966. Ce Comité est devenu en 2001, après plusieurs modifications, la Délégation générale à la langue française et aux langues de France et comprend une Commission générale de terminologie et de néologie. Le nouvel intitulé de cet organisme avec l'élargissement de ses attributions est intéressant, car il reconnaît l'existence, dans l'Hexagone, d'autres langues à côté du français. La tâche de ce Comité consiste à enrichir la langue et à développer son emploi.

L'enseignement favorisa la fixation et l'extension du français :

g) la création de l'école primaire sous la Révolution française, puis l'instauration de la gratuité et du caractère obligatoire de l'éducation avec les lois Jules Ferry (1881-1882), l'élaboration de manuels, de grammaires, de dictionnaires, etc., permirent sa diffusion.

h) Actuellement, le ministère de l'Éducation nationale exerce une régulation permanente de la langue avec des instructions officielles, un programme d'enseignement du français et l'instauration d'un corps d'inspecteurs vérifiant l'application de ce dernier.

Cette unification linguistique permit d'asseoir l'unité politique du pays; le français fut institué officiellement langue de la République française, en 1992 (cf. la modification de l'article 2 de la Constitution de la $\mathrm{V}^{\mathrm{e}}$ République).

Le français « officiel » est donc le résultat d'un long processus de formation continu, ininterrompu, qui a impliqué le pouvoir politique, l'aristocratie ainsi qu'une bourgeoisie dite «éclairée» et une certaine élite intellectuelle. Il a enfin été pris en charge par des institutions, avant d'être reconnu comme langue d'État.

D'abord langue du droit et de l'administration visant à faciliter la transmission et la compréhension des textes juridiques, le français devint très vite la langue de la Cour, des lettrés et des bourgeois avant de se généraliser. Le français « officiel » repose donc sur plusieurs usages, à la fois sociaux et géographiques, même si celui de la Cour et de l'aristocratie d'une part, et corrélativement de Paris et de l' ̂lle-deFrance d'autre part, semblent prédominants. Au cours de l'histoire, il revêtit une importance politique considérable dans l'unification du pays et la centralisation du pouvoir politique. Son caractère « officiel », sa référence aux textes littéraires largement privilégiés aux dépens des autres formes de la langue écrite et à plus forte raison de la langue orale, son souci du bon et du bel usage, prôné par la plupart des gens de lettres permirent de le valoriser en lui conférant un prestige social. L'intervention d'institutions pour son maintien dans une relative stabilité ainsi que pour son enrichissement et sa diffusion était et reste non seulement une 
garantie d'adaptation aux besoins de la société, toujours en évolution, mais aussi de protection de la langue elle-même vis-à-vis d'autres langues, de l'État et de ses citoyens.

\subsubsection{La norme du tchèque ${ }^{7}$}

L'établissement du tchèque « officiel » est, lui aussi, étroitement lié à l'histoire politique du pays et aux soubresauts que celui-ci a connus.

a) Le tchèque, ayant pour origine le protoslave, fut la langue «officielle » du Royaume tchèque, au $\mathrm{X}^{\mathrm{e}}$ siècle, sous la dynastie des Přzemysl.

b) Il coexistait partiellement avec l'allemand, puisque le Royaume, constitué par la Bohême, la Moravie et la Silésie, était peuplé non seulement de Tchèques, mais aussi d'Allemands.

c) La langue connut un véritable essor au XIV ${ }^{\mathrm{e}}$ siècle, sous le règne du Roi Charles IV et jusqu'au début du $X V^{\mathrm{e}}$. Le tchèque était utilisé dans l'administration politique et judiciaire, ainsi que comme langue littéraire, à côté du latin et de l'allemand.

d) C'est à cette époque aussi que Jan Hus (1371-1415), «ordonné prêtre en 1400 et Doyen de la Faculté de théologie de Prague l'année suivante » (Laran, cf. Encyclopaedia Universalis et http://www.museeprotestant.org), puis «élu recteur de l'Université de Prague en 1409»(Laran, ibid.) fut non seulement un réformateur religieux avant Luther, mais devint aussi, à travers ses prêches, l'un des grands défenseurs et diffuseurs de la langue tchèque. "L'Évangile est, selon lui, la seule règle infaillible et suffisante de la foi, et tout homme a le droit de l'étudier pour son propre compte ; il entreprend donc de le traduire en tchèque ${ }^{8}$, ce qui contribue à fixer la langue littéraire tchèque ». Il révisa plus tard sa traduction? Il s'occupa également de l'orthographe tchèque avec notamment l'introduction de signes diacritiques. L'ouvrage De orthographia bohemica (1410) qui vise à stabiliser la forme écrite lui est attribué avec une quasi-certitude selon plusieurs sources numériques (cf. entre autres :

http://data.bnf.fr/14415860/jan_hus_orthographia_bohemica/), alors que certains semblent considérer qu'il est anonyme.

En rébellion contre l'Église, Hus mourut sur le bûcher, ce qui provoqua une guerre politique, sociale et religieuse.

e) Le travail sur la langue de Jan Hus fut poursuivi par Jan Blahoslav. Ce dernier fut à l'origine d'une nouvelle traduction en tchèque du Nouveau Testament (1564) et joua un rôle majeur dans l'édition de la Bible de Kralice, considérée encore aujourd'hui comme un ouvrage majeur pour la langue et la culture tchèques. Parallèlement, il rédigea une grammaire tchèque, Gamatika česká (1571).

\footnotetext{
${ }^{7}$ Ce paragraphe est une synthèse d'informations extraites des ressources numériques mentionnées dans la bibliographie. Elles ont été comparées entre elles afin de vérifier autant que faire se peut leur validité.

${ }^{8}$ Cette information se retrouve sur le site du musée protestant (cf. bibliographie: ressources numériques).

${ }^{9}$ Cf. www.larousse.fr/encyclopedie/personnage/Jan Hus124504.
} 
f) Rattachées à la maison des Habsbourg en 1526, la Bohême et la Moravie furent profondément marquées par la guerre de Trente Ans (1618-1648), période appelée par les Tchèques «l'ère des ténèbres ». Elles connurent un triple affrontement, religieux, en raison du conflit entre les protestants et les catholiques, politique avec la confrontation entre la féodalité et l'absolutisme résultant de la soumission aux Habsbourg et linguistique.

En effet, sur le plan linguistique, la répression des protestants s'accompagna d'un véritable processus de germanisation (1621-1627) et par voie de conséquence d'un recul du tchèque au profit de l'allemand, qui devint langue officielle de la Bohême en 1784 :

«La langue nationale se voyait graduellement éliminée de toutes les sphères de la vie publique, de telle sorte que le tchèque semblait avoir périclité au point de n'être plus qu'une langue populaire » (MATHESIUS, $1947:$ 441-442).

Ce déclin de la langue tchèque, dont la fin fut marquée notamment en 1836 par la publication du poème Máj (Mai) de Mácha, fut clairement interrompu lors du renouveau national (1848-1918), qui vit le jour en réaction contre l'emprise germanique de plus en plus forte.

g) Le Printemps des Révolutions de 1848 manifesta une volonté de «restauration » et de réhabilitation de la langue, que l'on chercha à débarrasser des germanismes, une remise à l'honneur de la littérature et de la culture de l'époque, et, à travers cela, par le désir de faire revivre la conscience nationale, et de retrouver une identité propre. Parmi les intellectuels qui participèrent à ce mouvement, on trouve, entre autres, Josef Dobrovský, Josef Jungmann, František Palacký, disciple de Dobrovský, Karel Jaromír Erben, Pavel Jozef Šafárik, Hermenegild Jireček, Jan Gebauer, etc., qui, tous, publièrent une histoire, une grammaire ou un dictionnaire de la langue tchèque.

h) L'Académie tchèque pour les Sciences, les Lettres et les Arts fut fondée en 1891. Elle avait pour mission de publier des textes en vieux tchèque, notamment une collection de «Sources pour l'étude de la vie littéraire en Bohême, en Moravie et en Silésie ».

i) Ce retour aux sources exprimait non seulement un besoin de pureté et de protection du tchèque contre les agressions subies par la langue, mais aussi la volonté de faire renaître une identité nationale. Cette redécouverte de la langue et de la littérature eut nécessairement pour corollaire une certaine formalisation du tchèque.

j) C'est autour des années 1920-1930 que ce travail sur la langue fut entrepris et systématisé par les linguistes du Cercle de Prague sur des bases scientifiques dans le cadre de la théorie structurale et fonctionnelle dont ils furent les initiateurs.

Conscients tout d'abord, à l'instar de MATHESIUS, que

« le tchèque était «une langue au passé relativement récent qui, contrairement à d'autres, ne [s'était] pas développée à partir d'un dialecte de classe local mais par l'introduction d'une langue élaborée partiellement sur l'exemple d'une langue standard du passé, presque disparue, [...] artificielle dans sa morphologie archaïsante » (1932 : 14-31), 
ces chercheurs estimèrent nécessaire de mettre en place une nouvelle forme de langue standard plus adaptée à la vie quotidienne. L'élaboration de cette langue ne devait plus reposer sur son histoire, ni sur la recherche de sa pureté mais sur la description scientifique et objective du tchèque, fondée sur l'analyse du système dans son fonctionnement, avec la prise en compte de sa stabilité flexible. Mais il ne s'agissait pas pour autant de faire table rase du passé. Le point de vue synchronique s'étant substitué au point de vue diachronique, il convenait d'observer et de décrire les faits dans une perspective réaliste et fonctionnelle. Quant à l'usage retenu, " en l'absence d'une tradition châtiée de conversation tchèque » (MATHESIUS, 1932: 14-31), et compte tenu de l'objectif visé qui était d'atteindre «le plus haut niveau possible de développement fonctionnel de la langue, $[\ldots], \mathrm{d}^{\prime}[\mathrm{en}]$ parachever la spécificité nationale » et d'enrichir le patrimoine culturel du pays (MATHESIUS, 1932 : 14-3), le choix s'est porté sur « la pratique linguistique des bons auteurs tchèques, telle qu'elle se manifeste dans la moyenne de la littérature tchèque des cinquante dernières années, dans la littérature au sens le plus large du mot, tant celle des belles-lettres que celle de la science » (MATHESIUS, 1932 : 14-31). Le recours à la langue littéraire offrait, en outre, un usage relativement stable, ce que ne permettait pas le langage populaire avec ses variations dialectales. Néanmoins il s'avérait insuffisant pour satisfaire l'ensemble des besoins de la société. Le rôle du CLP fut donc aussi de pratiquer la culture de la langue au sens où l'a définie Havránek. Celle-ci implique en effet une «attention théorique consciente » dirigée « vers le perfectionnement et l'épanouissement de la langue standard » (HAVRÁNEK, 1932 : 32-84).

Le tchèque «officiel»s'est donc fait de façon discontinue. Après avoir été posé comme langue officielle par le pouvoir politique, il fut par la suite menacé par l'allemand, qui le relégua dans des usages populaires à l'extension limitée ; il devint alors l'objet d'interventions individuelles, hommes d'Église, grammairiens, philologues, etc., puis d'institutions scientifiques telles que le Cercle linguistique de Prague et l'Académie tchèque. Sa forme « officielle » actuelle, élaborée à partir de la langue littéraire de la période contemporaine du CLP est le résultat d'une action volontaire sur la langue provenant essentiellement des linguistes dont la tâche consistait à décrire, à codifier et à promouvoir le tchèque, de manière à élargir ses sphères d'emploi tout en lui conférant un prestige social puisque l'usage de référence était la langue écrite des bons auteurs. Le tchèque repose donc en grande partie sur la conscientisation et l'intellectualisation de la langue, censées favoriser une expression " juste, rigoureuse et abstraite », tout en couvrant l'ensemble des domaines de la vie quotidienne, l'une des fonctions essentielles étant de faire revivre la culture afin que les Tchèques puissent se réapproprier une identité nationale.

Sa diffusion repose sur les mêmes instances que celles du français, création de l'imprimerie, ministère de l'Éducation, de la Jeunesse et des Sports par le biais de l'enseignement, maisons d'édition avec l'élaboration de manuels, etc.

À l'heure actuelle, le tchèque n'est pas reconnu officiellement par la Constitution comme la langue de l'État, bien qu'il le soit implicitement, d'autant 
que la maîtrise de la langue est requise pour l'acquisition de la citoyenneté tchèque $^{10}$.

\subsubsection{Synthèse}

La mise en parallèle du français et du tchèque « officiels », fondés de façon plus ou moins prégnante sur la langue écrite, montre à quel point les critères d'établissement de la norme et les priorités (langue essentiellement littéraire ou non) diffèrent en fonction du contexte historico-politique de chacun des deux pays : en France, primauté de l'aspect politique au départ avec centralisation du pouvoir, en République tchèque, défense de la langue pour sa survie, en raison de conflits politiques et religieux, prééminence de l'aspect culturel et identitaire avec une très forte idéalisation de la langue et de la problématique de la codification. Néanmoins leurs fonctions respectives restent les mêmes, l'accent étant mis sur tel ou tel aspect selon le cas (unification de la langue, développement de l'intercompréhension, de l'éducation, transmission de la culture, instauration d'une identité nationale, renforcement du pouvoir politique, etc.).

En conclusion, on pourrait reprendre le propos de Bruno Migliorini (https:/fr.wikipedia.org/wiki/Histoire_de_la_langue_italienne) selon lequel «En France, l'unité politique a promu l'unité linguistique, alors qu'en Italie, l'unité linguistique a promu l'unité politique ${ }^{11}$, et j'ajouterai par comparaison avec la France qu'en République tchèque la survie et la renaissance de la langue tchèque ont été étroitement associées à celles de la culture et de l'identité tchèque au sein d'un pays qui a retrouvé parallèlement une unité politique. Ces remarques montrent clairement que les facteurs déclenchant l'instauration d'une norme varient considérablement d'un pays à l'autre, en fonction de leur situation respective, géopolitique, économique, linguistique, culturelle, etc., même si, lors de l'élaboration de la norme, ils sont susceptibles d'intervenir conjointement.

\subsection{Examen de différents points de vue}

\subsubsection{Frei}

Dans La grammaire des fautes (2007 [1929]), FREI aborde la question de la norme en référence à l'analyse des erreurs. Il part donc de l'opposition fonction vs norme et considère que les productions linguistiques n'ont pas à être évaluées d'un point de vue normatif mais d'un point de vue fonctionnel, le respect de la norme et celui de la fonction pouvant être en contradiction : par exemple, dans il l'a faite venir, l'accord au féminin de faire enfreint la norme, alors qu'il permet d'identifier le référent du pronom comme étant un mot féminin, contrairement à il l'a fait venir, la forme $l$ supprimant la distinction masculin/féminin.

\footnotetext{
${ }^{10} \mathrm{http}: / /$ www.axl.cefan.ulaval.ca/europe/reptcheque.htm, cf. § 4-4.1.

${ }^{11}$ Ce point de vue semble, en grande partie, partagé par Tullio de Mauro (1970) : «[...] la langue fût le symbole de la nation et [...] l'adhésion à ses normes fût une preuve de nationalité », cf. Florence Courriol (2013), Plurilinguisme littéraire et norme linguistique dans l'Italie contemporaine, Norme(s) et (a)normalité, Sciences Humaines Combinées, $\mathrm{n}^{\circ}$ 11, Revue électronique des écoles doctorales ED LISIT et ED LETS, p. 2.
} 
Le point de vue normatif repose sur une norme sociale «établie par la collectivité » (2007: 16) ; il est fondé sur des critères externes à la langue et implique un jugement de valeur. Il n'a aucune valeur explicative.

Le point de vue fonctionnel, au contraire, vise à analyser les faits en euxmêmes en référence à la fonction qu'ils assument. Il devrait donc primer sur le point de vue normatif dans l'explication des fautes.

\subsubsection{Hjelmslev}

Dans «Langue et parole » publié en 1943 (1971 : 77-89), HJELMSLEV propose une théorisation de la norme, sans référence à une langue quelconque. Jugeant, en effet, que la distinction entre la langue et la parole posée par Saussure est ambigüe, « chacun des deux termes [...] admettant des acceptions différentes » (1971: 79), HJELMSLEV établit une triple opposition, dont l'une met en jeu deux notions, l'usage en corrélation avec l'acte :

a) La norme, c'est-à-dire la langue sous sa «forme matérielle », " définie par une réalisation sociale donnée, mais indépendamment encore du détail de la manifestation » (1971:80). Elle découle de l'acte et de l'usage, dont elle est issue (1971 : 84). Quant à la manifestation, elle peut être d'ordre phonique, graphique, etc. $(1971: 81)$

b) L'usage, c'est-à-dire «l'ensemble des habitudes adoptées dans une société donnée et définies par les manifestations observées » (1971: 80). Cet usage s'actualise et se concrétise à travers des actes individuels.

c) Le schéma ou « forme pure », "définie indépendamment de sa réalisation sociale et de sa manifestation matérielle », qui correspond à la langue conçue comme une abstraction (1971:80).

Ces différentes notions sont en relation d'implication, soit bilatérale comme l'usage et l'acte qui se présupposent respectivement, soit unilatérale telle la norme qui repose sur l'usage et l'acte, et le schéma qui implique à la fois l'usage, l'acte et la norme comme le montre le schéma ci-dessous ${ }^{12}(1971: 86)$ :

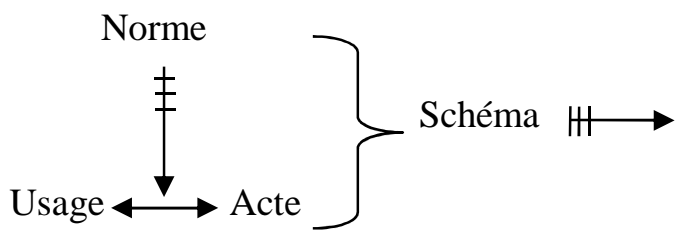

Fig. 1. Usage, Acte, Norme, Schéma (HJelmsLev, $1971: 86$ )

Norme, usage et acte sont solidaires, puisqu'ils ne peuvent exister l'un sans l'autre. Alors que l'usage fait l'objet de l'exécution, «la norme n'est en réalité qu'une construction artificielle, et l'acte d'autre part n'est qu'un document

\footnotetext{
${ }^{12}$ La flèche barrée indique l'implication, l'élément à la pointe de la flèche correspond à la constante et celui qui est placé à la base de la flèche barrée à la variable (1971:86).
} 
passager » (1971: 88). Seuls l'usage et l'acte renvoient à des réalités concrètes. La norme, étant une abstraction dégagée de l'usage, est une «fiction » (1971: 88).

\subsubsection{Coseriu}

Estimant, comme Hjelmslev, que la distinction langue/parole de Saussure est inadéquate et insuffisante, il propose, lui aussi, une tripartition du langage, relativement proche, dans les termes, de celle de Hjelmslev, mais différente dans la conception respective des notions retenues par l'un et par l'autre. Coseriu distingue donc le système, la norme et la parole ou le discours selon la traduction qui est faite de habla, ne faisant pas de distinction conceptuelle entre l'usage et l'acte.

Selon COSERIU, « La langue existe seulement dans et par la parole » (1958: 221). En conséquence, il s'avère impossible de concevoir le système tel qu'il a été envisagé par Saussure et repris par Hjelmslev, c'est-à-dire comme une forme et plus précisément comme une forme du contenu, indépendamment de la substance, puisque la langue, chez Coseriu, est envisagée à partir d'actes de parole concrets, lesquels priment sur le système.

Par rapport à la parole, et plus précisément à la réalisation concrète de l'acte de parler,

« la norme comprend tout ce qui [...] est traditionnellement (socialement) fixé, qui est usage commun et courant de la communauté linguistique [...]. Comme corollaire, la norme est un ensemble formalisé de réalisations traditionnelles ; elle comprend ce qui «existe » déjà, ce qui se trouve réalisé dans la tradition linguistique [...] » (COSERIU, 2001 : 246-247).

Elle peut donc inclure des variantes (cf. le [1] sonore en français qui correspond à la réalisation habituelle la plus fréquente et le [l] sourd que l'on rencontre à la finale d'un mot après une consonne sourde, comme dans peuple). La norme admet les deux réalisations. En revanche, elle se différencie du système, en ce que ce dernier n'est constitué que d'éléments fonctionnels pertinents. Il n'y aura, de ce fait, qu'une seule unité systémique /// par opposition à /t/, cf. /lap/ «lape » /tap/ «tape »:

« Le système $[\ldots]$ comprend tout ce qui est objectivement fonctionnel (distinctif). [...] le système est un ensemble de possibilités de réalisation : il comprend aussi ce qui n'a pas été réalisé, mais qui est virtuellement existant, ce qui est «possible » [...]» (CoserIU, $2001: 246$ 247).

Pour synthétiser son propos, Coseriu propose la schématisation suivante : 
A

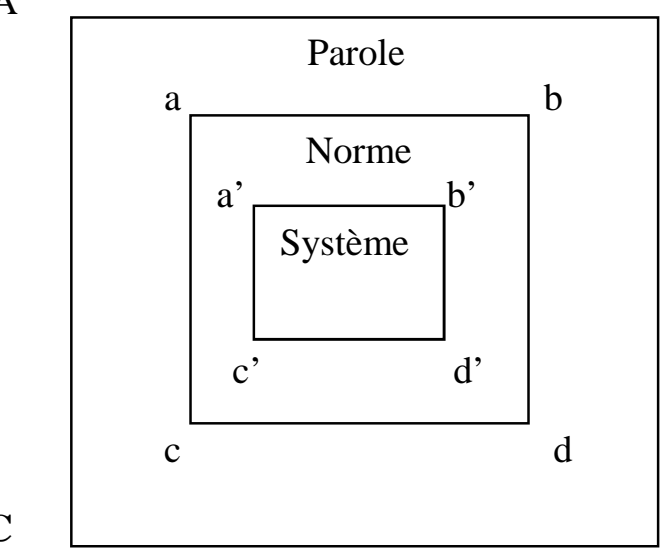

B

$\mathrm{D}$

Fig. 2. Parole, Norme, Système (CoSERIU, $1952: 95)^{13}$

Ce schéma met clairement en évidence le rapport d'inclusion de chacun de ces domaines et leur extension respective. La parole, au sens de réalisation langagière, représente le champ le plus large de l'analyse, à partir duquel sont construits la norme et le système, correspondant à un degré d'abstraction de plus en plus grand. Le système ne regroupe que les éléments fonctionnels de la langue alors que « la norme, c'est-à-dire la réalisation normale du système »(COSERIU, 1973 : 86-88) comporte non seulement les unités fonctionnelles systémiques mais aussi des variantes.

Ainsi conçue, la norme proposée se veut descriptive et objective mais non normative :

«[...] il ne s'agit pas de la norme au sens courant, établie ou imposée selon des critères de correction et de valorisation subjective sur ce qui est exprimé, mais de la norme objectivement vérifiable dans une langue; la norme que nous suivons nécessairement en tant que membres d'une communauté linguistique et non celle d'après laquelle il est reconnu que «nous parlons bien » ou de façon exemplaire, dans la communauté elle-même. En vérifiant la norme à laquelle nous nous référons, nous vérifions comment l'on dit, sans indiquer comment l'on doit dire » (COSERIU, 1973 (trad. JACQUET ANDRIEU, 1978 : 90)).

Fondée sur des usages communs et courants mais aussi traditionnellement fixés, elle relève à la fois de la synchronie et de la diachronie. Il s'agit donc non seulement d'un produit social dont les réalisations sont ou peuvent être attestées mais aussi d'un produit de l'histoire. Par ailleurs, elle a un double statut : « [elle] fait partie du bagage social que possèdent les sujets parlants » et « existe sur le plan psychique du langage »(GARVIN, 1983 : 14).

\footnotetext{
${ }^{13}$ Tableau repris dans Régis MissiRE (2004 : 16).
} 
Après avoir défini le concept de norme sur un plan général, Coseriu distingue donc à côté de la norme sociale une norme «individuelle » propre à un locuteur donné. Il dégage également des normes partielles, sociales, régionales, (COSERIU, 1952: 77), auxquelles s'ajoutent le langage familier, populaire, littéraire, soutenu, vulgaire, etc. (COSERIU, 2001: 98). Ces subdivisions sont rendues possibles par le fait «que la norme par sa nature même, est toujours moins générale que le système » (COSERIU, $1952: 77)$.

\subsubsection{Anne-Marie Houdebine}

Partant, comme Martinet, du principe que toute étude linguistique doit être à la fois descriptive et explicative, Anne-Marie Houdebine met clairement en évidence le fait que l'explication ne saurait reposer sur les seuls facteurs linguistiques, mais requiert le commentaire du sujet parlant sur ses productions. Elle a ainsi élaboré la théorie de l'Imaginaire linguistique, afin de rendre compte du regard du locuteur sur son propre discours dans l'analyse des données. C'est dans ce cadre-là qu'elle a développé le concept de norme, en s'inspirant de Hjelmslev, de Coseriu et de Rey (1972). Elle partage tout d'abord le point de vue de Hjelmslev, selon lequel la norme est une fiction. Notant par ailleurs que cette notion est polysémique, et qu'elle peut avoir soit une valeur descriptive et désigner un usage habituel, soit une valeur prescriptive et imposer un usage particulier, elle restreint, d'une certaine manière, cette polysémie en excluant la norme prescriptive du domaine linguistique. En revanche, elle l'élargit sur le plan descriptif en distinguant deux grands types de normes, les normes objectives et les normes subjectives qu'elle subdivise en plusieurs normes, toutes étant susceptibles d'interagir entre elles, comme le souligne le schéma :

a) Les normes objectives reposent sur l'analyse des productions des locuteurs, indépendamment $\mathrm{du}$ regard que ces derniers portent sur elles. Les normes objectives regroupent les normes statistiques et les normes systémiques. Les normes statistiques correspondent à des co-occurrences d'usages dans une langue donnée. Les normes systémiques sont dégagées à partir de l'étude d'un ou deux idiolectes $(2002: 20)$.

b) Les normes subjectives tiennent compte du rapport du locuteur à son discours. Elles comprennent les normes communicationnelles, qui visent «la compréhension, l'intégration » à un groupe particulier, les normes fictives qui renvoient à «un idéal de langue » personnel, même s'il peut être partagé par d'autres locuteurs et les normes prescriptives, qui réfèrent à un idéal de langue, souvent « puriste », institutionnalisé (cf. les grammaires scolaires) (2002:20).

Normes objectives et normes subjectives sont en rapport d'interaction : cellesci servent à éclairer et à mieux comprendre les usages des locuteurs, ainsi que leur variabilité : 


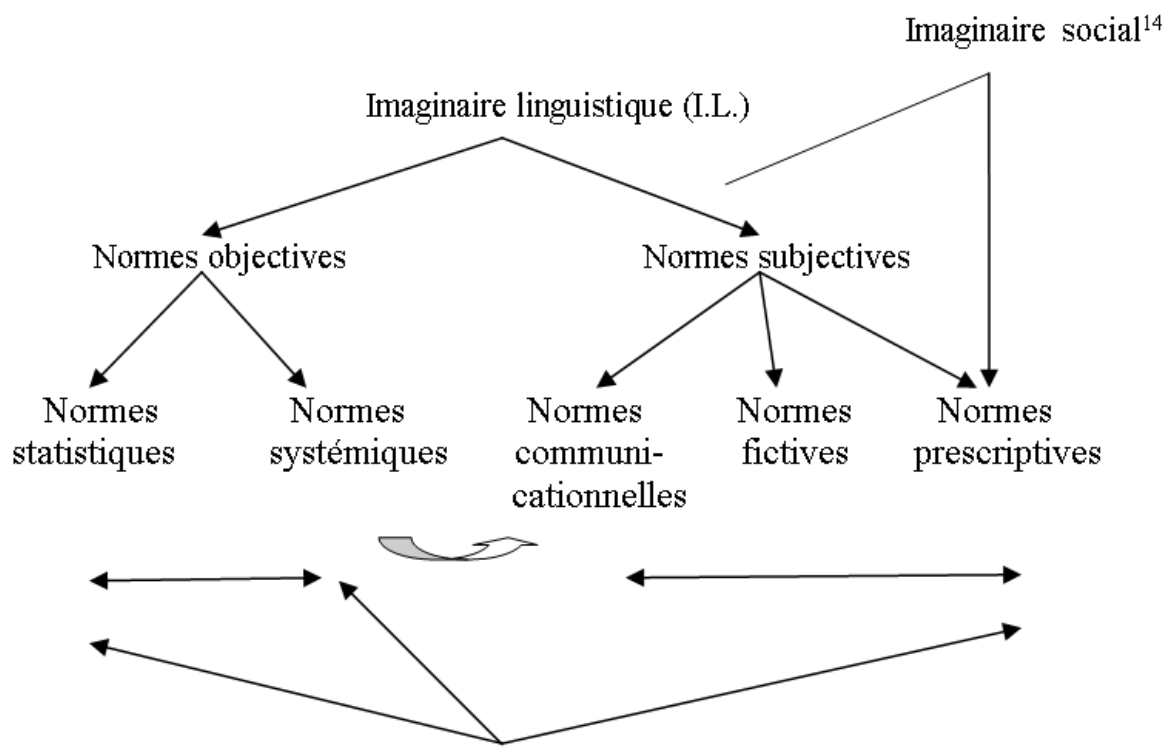

Fig. 3. L'Imaginaire linguistique - Interactions des diverses normes (HOUDEBINE, $2002: 21$ )

\subsubsection{Les membres du Cercle linguistique de Prague}

Comme l'affirme Ondřej Pešek,

« à l'instar de la phonologie, la linguistique comparative ou l'analyse de la perspective fonctionnelle de la phrase, la théorie de la norme et de la codification de la langue standard représente l'un des apports principaux de l'École de Prague » (PEŠEK, 2007 : 213).

Cette théorie de la norme se situe dans une perspective fort différente de celle des auteurs qui ont été mentionnés précédemment. Elle est indissociable de la mise en place d'une nouvelle norme "standard ${ }^{15}$ du tchèque (cf. § 2.2. 1. b) et se trouve par suite étroitement liée au statut et au rôle assignés à la langue dans le pays avant et après sa «codification ».

\section{Mathesius}

Partant du constat que la langue tchèque, après avoir été progressivement écartée de la vie publique, se reconstruisait essentiellement sur l'ancienne langue (MATHESIUS, 1948 : 441-442), Mathesius, en collaboration avec d'autres membres du CLP, notamment Havránek, et à un certain moment Weingart ${ }^{16}$, participa activement à l'élaboration d'une nouvelle norme «standard», comme nous l'avons vu précédemment. Aussi, seuls les principes fondamentaux sur lesquels

\footnotetext{
${ }^{14}$ La flèche est en pointillés dans le schéma d'origine.

${ }^{15}$ Le terme «standard » est provisoirement l'équivalent de «officiel ». Ce point sera discuté plus tard.

${ }^{16}$ Merci à l'un des relecteurs de cet article de m'avoir communiqué l'information.
} 
celle-ci doit s'appuyer seront rappelés, à savoir le respect de la stabilité flexible des langues s'accompagnant de la nécessité d'une régulation, qui garantissent l'une et l'autre la communication et la compréhension, auxquelles s'ajoutent le recours à la codification pour enrichir la langue et l'adapter aux besoins de la société.

\section{Havránek}

La codification obéit au principe de la culture consciente de la langue développé par Havránek. C'est l'un des critères qui permet à ce dernier d'opposer la langue «standard » à la langue populaire et à la langue urbaine commune.

a) La langue "standard» ne découle pas simplement de l'usage ; elle résulte de diverses interventions théoriques et scientifiques (CLP, Académie) et se caractérise par des fonctions multiples et variées, susceptibles de toucher l'ensemble des domaines de la vie quotidienne.

b) La langue populaire n'est pas codifiée. Elle ne met en jeu que l'usage et ses sphères d'emploi sont beaucoup plus limitées.

c) La langue urbaine commune, ou interdialecte, fait intervenir différents usages (parlers de la capitale, Prague, et des zones environnantes).

\section{Hausenblas}

S'inscrivant dans la lignée de Mathesius et de Havránek, Hausenblas considère que « la base de la culture de la langue réside dans la connaissance scientifique de la norme réelle de la langue littéraire » (GARVIN, 1983 : 4).

Contrairement à l'usage qui renvoie à ce qui est fréquent, habituel, abstraction faite du caractère correct ou non de l'emploi considéré, il estime que

« la notion de norme implique, en plus, un élément de conformité à des règles établies. La norme de la langue standard (les autres variétés de la langue nationale ayant, elles aussi, chacune leur norme), $[\ldots]$ est l'ensemble des règles ayant une existence objective et provenant de la compréhension mutuelle d'une collectivité qui sont perçues et acceptées comme obligatoires dans l'usage collectif des locuteurs d'une langue donnée »(1960:14-15).

Il oppose ainsi :

a) «La norme réelle qui se trouve dans la pratique littéraire et intellectuelle de la génération actuelle et de celle qui l'a immédiatement précédée »,

b) [et] la norme fictive [qui] réside dans les idées «romantiques » des puristes » (GARVIN, $1983: 4)$.

\subsubsection{Synthèse}

La confrontation des acceptions et des types de normes proposés témoigne de la diversité, parfois contradictoire, des points de vue adoptés :

Si l'on peut admettre d'une manière générale que la norme est conçue explicitement ou implicitement, c'est-à-dire sans que cela soit clairement affirmé, comme une construction sociale, «établie par la collectivité » ainsi que le dit Frei, elle renvoie néanmoins à deux approches en grande partie opposées, puisqu'elle est instituée tantôt comme 
a) une fiction (Hjelmslev, Houdebine), tantôt comme

b) une « réalité » linguistique dans la mesure où elle repose sur des données observables, censées être vérifiables (Coseriu, Mathesius, Havránek, Hausenblas), notamment à partir de la fréquence des éléments. Mais Coseriu introduit une perspective diachronique en estimant que la norme est traditionnellement fixée, alors que Mathesius, Havránek et Hausenblas privilégient la synchronie, envisagée d'un point de vue dynamique d'après le principe de la stabilité flexible de la langue. En d'autres termes, si fiction et «réalité » linguistique, dans la construction de la norme, mettent obligatoirement en jeu des faits langagiers issus de l'observation, dans la fiction, le poids de l'imaginaire conscient ou inconscient du descripteur et donc de sa subjectivité y joue un rôle beaucoup plus important que dans une approche qui se veut «réaliste », laquelle accorde la primauté à des critères présumés objectifs, tels que la fréquence.

Du reste, cette dichotomie n'est pas aussi nette que ce que l'on pourrait croire, dans la mesure où chez les promoteurs de la norme supposée « réelle » et plus précisément objective, on rencontre des normes dites fictives (Havránek, Hausenblas), et inversement chez d'autres chercheurs, qui se placent dans le domaine de la fiction, sont posées des normes objectives (Houdebine).

Cependant, il convient de souligner que Havránek et Hausenblas n'évoquent la norme fictive que pour la rejeter en raison de l'aspect puriste qu'ils lui confèrent.

On voit donc déjà que la frontière entre données observables et fiction est loin d'être étanche, ce qui pose un problème théorique et par suite méthodologique. Par ailleurs, la notion de norme fictive est particulièrement ambigüe, puisqu'une même appellation renvoie soit à une construction artificielle (Hjelmslev) mais ancrée en synchronie, soit au respect de la tradition, c'est-à-dire de l'histoire de la langue qui réfère à la diachronie (Havránek, Hausenblas), soit au regard que le locuteur est supposé porter sur son discours, ce dernier pouvant être envisagé comme affectif, esthétique, etc. (Houdebine).

D'autre part considérer que la norme est une construction sociale devrait exclure l'existence d'une norme individuelle (Coseriu, Houdebine), étant donné que celle-ci est examinée en référence au seul individu et non à la société, même si celle-ci l'influence nécessairement. La norme individuelle opposée à la norme sociale, contrairement à cette dernière, n'a une incidence sur la société que lorsqu'elle s'inscrit également, selon les usages, dans telle ou telle norme sociale.

En outre, si la norme est considérée comme une construction sociale, peut-elle avoir une réalité psychique (Coseriu) ? N'y a-t-il pas contradiction entre ces deux aspects ? On pourrait admettre, il est vrai, que cette construction sociale, une fois totalement intégrée par les individus qui l'adoptent acquiert une valeur psychique. Cependant cette présumée réalité psychique est soumise à un processus d'acquisition lié à la vie sociale. Elle n'est en aucun cas innée, ni inhérente à la norme. Il importe donc de bien hiérarchiser l'aspect social de la norme qui prime nécessairement sur son caractère psychique.

Le terme social est lui aussi ambivalent, car il est utilisé pour caractériser non seulement les acteurs à l'origine de la norme, à savoir la société, mais aussi, pour permettre, dans le cas d'une norme dite sociale (Coseriu), de délimiter au sein de 
cette société un sous-groupe particulier supposé relativement homogène sur le plan social qui se démarque des autres sous-groupes par des traits linguistiques censés leur être communs, tout en leur étant spécifiques, et de ce fait discriminants.

Quant aux relations conçues entre les notions de norme, usage/acte, schéma (Hjelmslev), et parole, norme, système (Coseriu), elles sont de nature opposée, les premières impliquant un rapport d'implication bilatérale (usage $\leftrightarrow$ acte) ou unilatérale (norme $\rightarrow$ usage/acte, et cet ensemble vis-à-vis du schéma, cf. § 3.1.2.), alors que les secondes se trouvent dans un rapport d'inclusion (cf. $\S$ 3.1.3.).

En revanche, la démarche suivie pour établir une norme est la même chez l'ensemble des auteurs. On peut la juger comme étant ascendante, puisqu'elle part des usages pour aller vers la norme et en définitive vers le système. Elle est, toutefois, élargie par Anne-Marie Houdebine, qui inclut, dans l'analyse, le regard des locuteurs sur leurs productions à travers leurs propres commentaires.

Les critères de détermination des normes sont aussi globalement les mêmes : la priorité est accordée à la fréquence chez Coseriu et chez Houdebine, malgré son caractère aléatoire, dû au fait qu'elle est susceptible de varier en fonction des locuteurs et des situations de communication (cf. § 3.1.3.). Un autre critère tout aussi fluctuant est celui du choix des usages pris en compte comme fondement de la norme : alors que Coseriu se centre au départ sur «l'usage commun et courant de la communauté linguistique » (cf. § 3.1.3.) Mathesius, Havránek et Hausenblas privilégient les textes littéraires au sens large du terme, afin que le tchèque standard puisse atteindre un haut niveau de développement linguistique (§ 3.1.5.). Hausenblas insiste sur un autre critère, qui me parait fondamental, celui de la mise en évidence de règles.

Coseriu adopte donc une position que je qualifierais de «non marquée », c'està-dire de «relativement neutre » contrairement aux linguistes tchèques, qui, tout en se situant au niveau de l'observation de données linguistiques, sélectionnent un usage marqué qui reflète une vision idéalisée et idéaliste de la langue.

Toutefois, il convient de rappeler que, face à la multiplicité des normes dégagées, les usages de référence varient nécessairement selon les normes établies; ils ne seront évidemment pas les mêmes dans le cas d'une norme dite populaire et dans celui d'une norme «standard».

\subsection{Examen des critères à la base de l'établissement des normes ${ }^{17}$}

Ce qui ressort de cette présentation des normes, hormis les normes « officielle » et «standard» que nous examinerons ci-après, c'est le caractère hétéroclite et disparate de leur dénomination et l'hétérogénéité des critères sur lesquels repose leur discrimination, comme l'illustre la liste cidessous :

1/ La référence à un usage institutionnalisé : la norme «standard » que j'ai qualifiée personnellement de « norme officielle» (cf. § 3.3.)

\footnotetext{
${ }^{17}$ Il se limite à celui des textes qui ont servi à étudier les auteurs considérés. Mais il est loin d'être exhaustif, la plupart des linguistes posant eux-mêmes leur propres normes et par voie de conséquence leur terminologie en fonction de l'objectif qu'ils assignent à leurs études.
} 
2/ Le critère qualitatif étayé par le critère quantitatif : les normes objectives, et plus précisément les normes statistiques

3/ Le critère qualitatif reposant sur l'évaluation subjective de certains usages par le descripteur : langage familier, soutenu, vulgaire (Coseriu).

4/ La structure de la langue : la norme systémique.

$5 /$ La référence au locuteur : les normes subjectives (Houdebine)

6/ La finalité attribuée par le locuteur à son discours, telle qu'elle est conçue par Anne-Marie Houdebine, cf. les normes communicationnelle, fictive et prescriptive.

7/ La contrainte d'emploi imposée par une autorité extérieure à la langue : la norme prescriptive, conformément à sa définition habituelle.

8/ L'appartenance ou non des locuteurs à un sous-groupe social : les normes sociales les normes individuelles, mais aussi les normes populaires, normes sociales et populaires impliquant de surcroît un jugement de valeur.

9/ Les zones d'emploi, la localisation: les normes régionales, les normes urbaines, dont la dénomination occulte leurs caractéristiques linguistiques.

10/ Le registre de langue qui présuppose aussi une évaluation : la norme littéraire.

Face à cette multiplicité des normes et des critères utilisés pour les différencier, la question qui se pose est de savoir d'une part si l'ensemble de ces critères sont discriminants, d'autre part si ces normes constituent des minisystèmes qui seraient exclusifs les uns des autres, ou si dans de nombreux cas, il ne pourrait pas y avoir intersection, voire superposition de certains d'entre eux, de telle sorte que ce serait, en définitive, le point de vue choisi par le descripteur et la visée de l'étude qui déterminerait l'intégration de tel usage à une norme plutôt qu'à une autre et non les données linguistiques elles-mêmes qui en seraient à l'origine. La fréquence, par exemple, n'est pas spécifique à une norme particulière, elle est l'une des conditions nécessaires à l'établissement d'une norme, quelle qu'elle soit. Par ailleurs, une expression telle que il fait vent, calquée peut-être sur il fait froid, mais plus proche structurellement de il fait jour, vent étant un nom (cf. jour) et non un adjectif (froid) relève-t-elle d'une norme régionale, sociale, populaire, etc.? Les trois hypothèses peuvent parfaitement se justifier. Si elle est utilisée par une majorité de locuteurs d'une région donnée, indépendamment de leur appartenance à un groupe social déterminé, on pourrait estimer qu'elle relève d'une norme régionale, mais cela nécessite une très large enquête. De plus, les mouvements de population au sein d'un territoire entraînent des mélanges linguistiques qui rendent l'analyse délicate. En revanche, si cet usage est aussi lié à une classe sociale particulière, et, de surcroît, populaire, le choix reste ouvert, les trois possibilités pouvant être retenues. Le choix de l'une d'elles aux dépens des deux autres est arbitraire du point de vue linguistique. Ces classifications ne reposent pas sur les données elles-mêmes, elles sont imposées et (ré)organisées par la vision du descripteur et les besoins de son étude. 


\subsection{Comparaison des différentes normes}

Qu'en est-il désormais de la norme dite «officielle» et de la norme « standard»? S'agit-il de deux normes différentes ou d'une même norme, dont la dénomination est simplement variable ? Rien ne permet véritablement de répondre à la question et l'on se trouve une fois de plus confronté au problème de la terminologie. Personnellement, je préférerais les dissocier. La norme « officielle » serait instituée par une ou plusieurs instances reconnues par l'État et promulguée comme telle par ce dernier, même si elle n'est pas nécessairement inscrite dans la constitution (cf. le tchèque dit «standard »). En revanche, plutôt que de poser une norme «standard», (cf. la langue des médias, par exemple), il me semblerait préférable de parler d'usage «standard» de la langue qui s'appuierait sur la «norme officielle» tout en intégrant des éléments nouveaux, non encore enregistrés par cette dernière. En d'autres termes, l'usage «standard» serait second et avant-gardiste par rapport à la norme "officielle», fondatrice et plus conservatrice, ce qui n'implique pas pour autant que celle-ci soit figée, son évolution se faisant rétroactivement par l'intermédiaire de l'usage « standard ».

Compte tenu de son caractère institutionnel et de son statut de langue d'État reconnu explicitement (cf. le français) ou implicitement (cf. le tchèque), la norme « officielle » sert de forme de référence à partir de laquelle les autres normes sont posées, contrairement à celles-ci. En outre, elle se différencie des normes précédemment mentionnées par ses caractéristiques, sa plurifonctionnalité, une extension d'emploi maximum et sa finalité, comme le résume le tableau cidessous : 


\begin{tabular}{|c|c|c|c|}
\hline & & $\begin{array}{c}\text { Norme } \\
\text { « officielle » }\end{array}$ & Autres normes \\
\hline Finalité & $\begin{array}{c}\text { Unification d'un territoire linguistique } \\
\text { statutaire }\end{array}$ & + & - \\
\hline \multirow{3}{*}{ Caractéristiques } & $\begin{array}{l}\text { Validité générale au sein de la } \\
\text { communauté étatique }\end{array}$ & + & - \\
\hline & $\begin{array}{c}\text { Adaptation continue, généralement } \\
\text { différée dans le temps par rapport } \\
\text { à l'usage }\end{array}$ & + & $(?)^{18}$ \\
\hline & Capacité à se développer & + & (?) \\
\hline \multirow{6}{*}{ Fonctions } & Stabilisation de la langue & + & - \\
\hline & $\begin{array}{c}\text { Unification et stabilisation du pouvoir } \\
\text { politique }\end{array}$ & + & - \\
\hline & $\begin{array}{l}\text { Généralisation de la compréhension } \\
\text { Facilitation de la communication }\end{array}$ & + & $\begin{array}{l}(+) \\
\text { éventuellement dans } \\
\text { un sous-groupe }\end{array}$ \\
\hline & $\begin{array}{c}\text { Couverture de l'ensemble des besoins } \\
\text { communicatifs (dont transmission de } \\
\text { l'éducation et de la culture) }\end{array}$ & + & - \\
\hline & $\begin{array}{c}\text { Protection (de la langue et des } \\
\text { locuteurs vis-à-vis d'autres langues) }\end{array}$ & + & - \\
\hline & $\begin{array}{l}\text { Valorisation (de la langue par rapport } \\
\text { à d'autres langues, et des locuteurs : } \\
\text { intégration, ascension sociale, etc.) }\end{array}$ & + & $\stackrel{(+)}{\text { dans certains cas }}$ \\
\hline
\end{tabular}

Tableau 1. Comparaison de la norme « officielle » et des autres normes

La norme « officielle» devrait être différenciée de ce que certains appellent la codification. Ce terme désigne prioritairement le processus de mise en forme de certains usages par la sélection et l'organisation d'éléments empruntés à ces derniers ; corrélativement, il peut renvoyer au résultat, mais alors mise en forme et résultat ne sont pas nettement dissociés. Or la norme est précisément le résultat de ce processus et le terme évite ainsi toute ambiguïté.

De plus, comme le fait apparaître le tableau, la norme «officielle» n'a quasiment aucun trait commun avec les autres normes.

Par ailleurs, bien qu'elle soit fixée à un moment donné, elle reste ouverte, dynamique et non totalement repliée sur les usages dont elle est issue. Le fait qu'elle exerce une fonction de protection ne lui confère aucunement un caractère xénophobe ou impérialiste comme on a pu parfois le lui reprocher.

\footnotetext{
${ }^{18}$ Le point d'interrogation signale l'incertitude et éventuellement la variabilité de comportement des différentes normes.
} 


\begin{tabular}{|c|c|c|c|c|c|}
\hline \multirow[t]{2}{*}{ Caractéristiques } & \multicolumn{3}{|c|}{ Normes non linguistiques } & \multicolumn{2}{|c|}{ Normes linguistiques } \\
\hline & industrielles & $\begin{array}{l}\text { esthétiques } \\
\text { (picturales) }\end{array}$ & $\begin{array}{l}\text { savoir- } \\
\text { vivre }\end{array}$ & « officielle » & autres \\
\hline $\begin{array}{l}\text { Formalisation } \\
\text { par l'intervention } \\
\text { d'une institution } \\
\text { ou d'un } \\
\text { organisme, etc. } \\
\text { (et non d'un } \\
\text { mouvement ou } \\
\text { d'un sous- } \\
\text { groupe) } \\
\end{array}$ & + & - & - & + & - \\
\hline Finalité objective & + & $\begin{array}{l}+ \\
\text { (différenciation } \\
\text { d'un } \\
\text { mouvement par } \\
\text { rapport à un } \\
\text { autre) } \\
\end{array}$ & $\begin{array}{l}\text { - } \\
\text { (finalité } \\
\text { subjective } \\
\text { et donc } \\
\text { fictive) }\end{array}$ & + & - \\
\hline Protection & + & + & - (idem) & + & - \\
\hline Valorisation & + & + & - (idem) & + & - \\
\hline $\begin{array}{l}\text { Impérative } \\
\text { (obligation de la } \\
\text { respecter) }\end{array}$ & + & - & - & $\begin{array}{c}? \\
\text { Fonction des } \\
\text { situations : } \\
+ \text { (concours, } \\
\text { etc.) } \\
\text { - (relative } \\
\text { liberté de choix } \\
\text { d'autres usages } \\
\text { (cf. certains } \\
\text { types de } \\
\text { correspondance) }\end{array}$ & - \\
\hline
\end{tabular}

Tableau 2. Mise en parallèle des normes non linguistiques et des normes linguistiques

Cette confrontation des normes montre une fois de plus que la norme « officielle » se singularise par rapport aux autres normes, mais qu'elle partage, en revanche, toutes les propriétés des normes industrielles, à un degré moindre cependant pour ce qui est du caractère impératif. Cela s'explique par une très grande variété d'usages à la disposition du locuteur qui échappent partiellement à la norme « officielle », variété qui s'oppose à l'unicité des principes imposés par la norme industrielle. Les soi-disant règles de bienséance ne forment pas une norme au sens strict du terme, car elles sont édictées par un ou plusieurs individus, sous prétexte qu'elles répondent à un certain savoir-vivre choisi de façon totalement subjective, sans aucune justification. Quant aux normes picturales, elles ont en quelque sorte un statut intermédiaire entre la norme linguistique dite «officielle» et les autres du fait qu'elle caractérise un mouvement au sein de la communauté des peintres et non l'ensemble des artistes.

La norme « officielle» comme les normes industrielles, plurielles par nature, puisqu'elles s'appliquent chacune à un domaine particulier, contrairement à la première, répondent ainsi à la définition initiale de la norme, dans la mesure où il 
s'agit au départ d'un produit, résultat d'une construction, qui impose des règles et devient ensuite un modèle découlant des caractéristiques qui lui sont attribuées. Ce modèle peut acquérir, de ce fait, un caractère impératif, voire prescriptif. Dans le cas de la langue, cet aspect impératif de la norme «officielle» est loin d'être absolu. Il est étroitement lié à son emploi dans des contextes déterminés, en principe hautement formalisés (cf. l'administration, l'enseignement, etc.). Cette adhésion ou ce manquement à la norme «officielle» par le locuteur est susceptible alors d'entraîner des sanctions, positives ou négatives (cf. une bonne ou une mauvaise note à une dictée). Mais on peut la transgresser sciemment ou non, pour des raisons diverses, désir de différenciation, révolte à l'égard de la société ou tout simplement maîtrise insuffisante de la langue et/ou méconnaissance de la forme que certains usages doivent prendre dans des contextes déterminés. Le caractère prescriptif de la norme «officielle » lui est conféré par une instance extérieure à la langue. Il n'existe pas de norme prescriptive qui s'opposerait à une norme « officielle».

La norme « officielle » répond ainsi parfaitement, en tant que modèle institué, à la définition du gnomon posée par BLAY (2007 : 568) : il s'agit, en effet d'un «principe de surveillance de la rectitude ou régularité d'une action ou d'un jugement » et j'ajouterai : dans une situation donnée. Mais, comme nous l'avons mentionné précédemment, cette norme « officielle » n'est pas figée ; elle doit tenir compte de l'évolution des usages, ce qui a pour conséquence l'intégration d'emprunts dans la langue (cf. leader au $\mathrm{XIX}^{\mathrm{e}}$ siècle) ou de créations (cf. mémorisable, pérenniser), etc.

L'élaboration de la norme «officielle» est présentée dans le schéma cidessous. Tout d'abord il convient de dissocier le circuit linguistique et le circuit institutionnel.

Le circuit linguistique comporte les éléments constitutifs de cette norme : du point de vue linguistique sont distingués le système, les usages et les actes, sans que ne soit présupposée une antériorité hiérarchique d'une entité par rapport aux autres. Système, usages, actes sont au départ mis sur le même plan. Du point de vue des acteurs impliqués dans ce circuit sont différenciés le locuteur (L), usager de la langue, et le descripteur (D), constructeur du système linguistique que l'on peut considérer trivialement comme le stock linguistique dans lequel le locuteur puise les éléments qui constituent ses actes de parole. Le locuteur part du système dont il active une partie des éléments dans ses productions. Ces dernières s'inscrivent dans des usages qui eux-mêmes renvoient au système. Le descripteur adopte la démarche inverse : il part des actes du locuteur qui relèvent de certains usages, et c'est à partir de ceux-ci qu'il élabore le système.

Le circuit institutionnel (I) intervient sur la langue, à la fois par la sélection d'éléments linguistiques issus de certains usages, par l'intégration de nouveaux éléments et l'enrichissement du système et par la diffusion. Cette «codification » que je préfère appeler normativisation, terme qui supprime ou atténue le caractère rigide, voire figé en dehors de toute intervention extérieure volontaire, impliqué par celui de codification, a une triple incidence, sur le système d'une part, sur les actes et sur les usages d'autre part, l'hypothèse étant que cette interaction entre la norme, le système, les usages et les actes est simultanée. 
B/ Circuit institutionne ${ }^{1}$

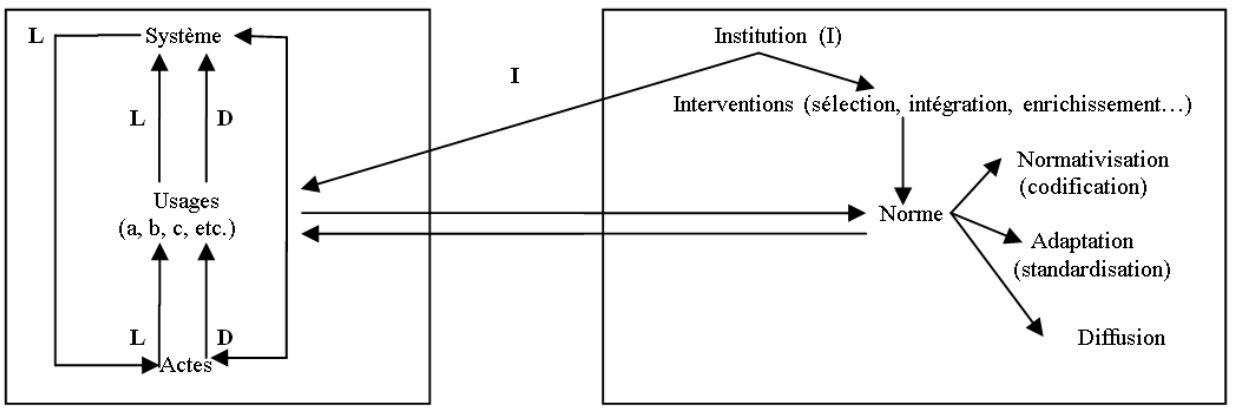

Fig. 4. Norme « officielle $»^{19}$ (Feuillard)

$\mathrm{L}=$ Locuteur, $\mathrm{D}=$ Descripteur, $\mathrm{I}=$ Institution

Comme le fait apparaître le schéma, la norme «officielle» est une construction conventionnelle, plutôt que fictive ou simplement arbitraire. À ce titre, tout en étant constituée d'éléments linguistiques, elle est, au départ, extérieure à la langue : elle exige un consensus de la part des concepteurs auquel doivent adhérer en principe les usagers, même si certains cherchent à la violer, soit par réaction contre le système politico-social, soit par un souci de différenciation et par une volonté d'affirmation identitaire, personnelle ou de groupe. Actuellement, elle s'appuie sur des usages essentiellement écrits, alors que la langue orale est à tort reléguée au second plan ; elle s'inscrit dans une synchronie dynamique (cf. adaptation), repose sur la fréquence d'emploi des éléments linguistiques et implique des règles de fonctionnement. Mais une fois établie, ses éléments constitutifs sont inclus dans le système et participent à son évolution, qui, à un moment donné, est formalisée par la standardisation. La norme «officielle » est donc nécessairement conscientisée et intellectualisée, ainsi que l'affirment Mathesius, Havránek et Hausenblas. Cela montre l'importance méthodologique de la notion de culture de la langue, totalement absente de la tradition linguistique française.

\subsection{Conclusion}

La norme « officielle » se distingue donc fondamentalement des autres normes, lesquelles entrent uniquement dans le circuit linguistique (A). Elle s'oppose à celles-ci notamment par son instauration volontaire, sa construction institutionnalisée et sa nature conventionnelle. Sa flexibilité et donc sa dynamique est liée à des facteurs à la fois internes et externes aux usages langagiers des locuteurs. En effet, certaines variations d'usage sont intégrées à la norme, alors que d'autres sont créées, en particulier par l'institution, impliquant un processus d'intellectualisation (cf. les problèmes de terminologie scientifique par exemple)

\footnotetext{
${ }^{19}$ Ce schéma ne prend pas en compte les facteurs situationnels qui interviennent dans les choix linguistiques.
} 
Les normes "non officielles", en revanche, peuvent être définies comme le regroupement d'un ensemble d'habitudes langagières convergentes effectué par un descripteur particulier, selon un point de vue qu'il a lui-même posé (sociologique, géographique, etc.), d'où leur grande diversité. Ces habitudes correspondent à des usages spécifiques, collectifs ou individuels et le point de convergence à partir duquel ces normes sont identifiées est déterminé en fonction de l'optique sélectionnée. Comme la norme " officielle », ces normes ne sont pas figées, mais leurs variations dépendent des usages des locuteurs censés les partager et du regard que porte le descripteur sur ces usages (cf. il fait vent). Par ailleurs, les groupes d'usagers de ces différentes normes peuvent être relativement fluctuants, dans la mesure où les locuteurs sont eux-mêmes multiples et changeants tant sur le plan linguistique qu'extra-linguistique (cf. maîtrise éventuelle de plusieurs registres et niveaux de langue, variations en fonction des situations de communication, changement de statut social, de lieu de résidence, etc.).

La mise en parallèle de ces deux types de normes fait clairement apparaître un glissement conceptuel fondamental de la notion première de norme par rapport à son acception initiale à laquelle réfère la norme «officielle », d'où la nécessité de spécifier le terme norme pour lever l'ambiguité (cf. norme urbaine, norme familière, etc.) et conférer à ce dernier une valeur opératoire dans le contexte où il est utilisé.

Les normes «non officielles » fondées sur la description ont une fonction de caractérisation et donc de différenciation, voire de discrimination. Elles permettent de délimiter et d'identifier un sous-groupe ou de spécifier certains usages d'un individu au sein d'une communauté linguistique donnée. De ce fait, elles peuvent avoir une incidence au niveau de l'explication dans la mesure où elles sont susceptibles de justifier certaines données langagières par opposition à d'autres.

Considérée individuellement, chacune de ces normes ne concerne qu'une partie de la collectivité, mais en aucun cas sa totalité, ce qui n'exclut pas qu'à terme, elle puisse influer sur la norme «officielle».

La norme "officielle », quant à elle et telle qu'elle vient d'être envisagée n'a, en soi, aucune valeur descriptive, puisqu' au départ, elle n'est pas inhérente à la langue; elle résulte d'une «construction». Néanmoins, les éléments qui la constituent sont nécessairement pertinents du point de vue linguistique, étant donné qu'ils sont intégrés au système et qu'ils le conditionnent rétroactivement (cf. le schéma). La norme « officielle», en permettant d'établir et de circonscrire un usage que l'institution cherche à généraliser, a une spécificité fonctionnelle propre, quelles que soient les langues considérées, qui est l'unification linguistique d'un pays (État). Elle se veut donc un facteur d'organisation, de structuration et de cohésion, pour reprendre certaines des caractéristiques mentionnées par Durkheim dans sa description des normes sociales examinées d'un point de vue global (PILLON, 2003 : 10). En conséquence, la prise en compte de la norme «officielle » par les locuteurs natifs ou non de la langue considérée contribue à leur intégration dans la société qui la pratique. 
Il apparaît ainsi que La norme "officielle », lorsqu'elle est instituée, détermine, en partie l'usage, mais la mise en évidence de "normes non officielles » tout comme l'apparition, entre autres, de nouvelles formes langagières grâce à l'emploi de nouvelles technologies (tchat, SMS, etc.) montre cependant que l'usage, au sens général du terme, conserve une partielle autonomie due à la flexibilité du système et donc à la souplesse que la langue laisse au locuteur dans l'acte de communication et dans son adaptation à la situation.

\section{RÉFÉRENCES BIBLIOGRAPHIQUES}

BLAY Michel (sous la dir. de) (2007 [2006]), Dictionnaire des concepts philosophiques, Paris, Larousse, CNRS éd.

COSERIU Eugenio (1958), Sincronia, diacronia e historia. El problema del cambio lingüistico, Montevideo, Facultad de humanidades y ciencias.

COSERIU Eugenio (1973 [1952]), Sistema, Norma y Habla, Teoria del lenguaje y Lingüistica general, Madrid, Gredos.

COSERIU Eugenio (2001), L'homme et son langage, Louvain, Peeters, Bibliothèque de l'information grammaticale, $\mathrm{n}^{\circ} 46$.

COURRIOL Florence (2013), Plurilinguisme littéraire et norme linguistique dans l'Italie contemporaine, Norme(s) et (a)normalité, Sciences Humaines Combinées, $\mathrm{n}^{\circ} 11$, Revue électronique des écoles doctorales ED LISIT et ED LETS.

DE MAURO Tullio (1970), Storia linguistica dell'Italia unita, Bari, Laterza.

FREI Henri (2007 [1929]), La grammaire des fautes, Rennes, Ennoïa.

GARVIN Paul L. (1983), Le rôle des linguistes de l'École de Prague dans le développement de la norme linguistique tchèque, in : BÉRARD Édith et Jacques MORAIS, éds., La norme linguistique, Conseil supérieur de la langue française, Québec.

HAUSENBLAS Karel (1960), O jazykové kultuře (De la culture de la langue), in : $O$ češtině pro Čechy (Le tchèque pour les Tchèques), Jazyková přríručka (Manuel linguistique), Novinářský studijní ústav (Institut d'études journalistiques), Prague, Orbis, p. 5-17.

HAVRÁNEK Bohuslav (1932), Úkoly spisovného jazyka a jeho kultura, in: Bohuslav HAVRÁNEK et Miloš WEINGART, (sous la direction de), Spisovná čeština a jazyková kultura (Le tchèque standard et la culture de la langue), Cercle linguistique de Prague, Prague, Melantrich, p. 32-84, traduit par Paul L. Garvin.

HJELMSLEV Louis (1968 [1943]), Prolégomènes à une théorie du langage, Paris, éd. de Minuit.

HJELMSLEV Louis (1971 [1959]), Essais linguistiques, Paris, éd. de Minuit.

HOUDEBINE-GRAVAUD Anne-Marie (2002), L'imaginaire linguistique : un niveau d'analyse et un point de vue théorique, in: Anne-Marie HoudEBINEGRAVAUD, (sous la dir. de), L'imaginaire linguistique, Paris, L'Harmattan.

JACQUET-ANDRIEU (1978 [trad. de E. Coseriu, 1973]), Système, Norme et Parole : présentation bilingue annotée, Mémoire de maîtrise, Université Lyon 2. 
LARAN Michel, Hus Jan (1370 env.-1415), Encyclopaedia Universalis (en ligne : <http://www.universalis.fr/encyclopedie/jan-hus/ >).

MATHESIUS Vilém (1932), O požadavku stability ve spisovném jazyce (Sur la nécessité de la stabilité d'une langue standard), in : Bohuslav HAVRÁNEK et Miloš WeIngart (sous la direction de), Spisovná čeština a jazyková kultura (Le tchèque standard et la culture de la langue), Prague, Melantrich, p. 14-31, traduit par Paul L. Garvin.

MATHESIUS Vilém (1947), Problémy české kultury jazykové (Problème de la culture de la langue tchèque), in: Vilém Mathesius, Čeština a obecný jazykozpyt (Le tchèque et la linguistique générale), Prague, Melantrich, p. 436458.

Migliorini Bruno (1966), Storia della lingua italiana, Firenze, Sansoni.

MisSIRE Régis (2004), Norme(s) linguistique(s) et afférence sémantique : une lecture de Sémantique interprétative à partir d'Eugenio Coseriu. Texto!, $<$ http://www.revue-texto.net/index.php?id=581>.

PEŠEK Ondřej (2007), Dynamique, norme et codification - qu'en est-il aujourd'hui de la théorie pragoise ?, in: Anna BochnaKowa, Agnieszka MARduŁA, Teresa TOMASZKIEWICZ (éds.), Norme, normativité, transgression, Oficyna Wydawnicza LEKSEM, Łask.

PILLON Véronique (2003), Normes et déviances, col. Thèmes et Débats, Paris, Bréal.

REY Alain (1972), Usages, jugements et prescriptions linguistiques, Langue française, vol. 16, $\mathrm{n}^{\circ}$ 16, Paris, Larousse.

\section{Ressources numériques}

http://www.radio.cz/fr/rubrique/literature/la-bible-de-kralice-fete-ses-400-ans http://www.axl.cefan.ulaval.ca/europe/reptcheque.htm http://www.cslf.gouv.qc.ca/bibliotheque-virtuelle/publication $\mathrm{html} /$ ?tx_iggcpplus_pi4\%5Bfile\%5D=publications /pubf101/f101p1.html http://www.revue-texto.net/Inedits/Missire/Missire_Normes.html. http://www.universalis.fr/encyclopedie/jan-hus http://www.universalis.fr/encyclopedie/jan-hus/1-le-reformateur/ http://www.larousse.fr/encyclopedie/personnage/Jan Hus124504 http :www.museeprotestant.org http://data.bnf.fr/14415860/jan_hus_orthographia_bohemica/ http://revuesshs.u-bourgogne.fr/lisit491/document.php?id=1014 https://fr.wikipedia.org/wiki/Histoire_de_la_langue_italienne 